\title{
ENFERMAGEM OBSTÉTRICA E SUA FORÇA DE TRABALHO EM TEMPOS DE COVID-19: RELATO DE EXPERIÊNCIA DAS REGIÕES DO BRASIL
}

Valdecyr Herdy Alves ${ }^{1}$

Kleyde Ventura de Souza²

Juliana Maria Almeida do Carmo ${ }^{3}$

Virginia Leismann Moretto ${ }^{4}$

Renata Cristina Teixeira ${ }^{5}$

Waglânia de Mendonça Faustino e Freitas ${ }^{6}$

Elisanete de Lourdes Carvalho de Sousa ${ }^{7}$ http://orcid.org/0000-0001-8671-5063 https://orcid.org/0000-0002-0971-1701 https://orcid.org/0000-0003-1572-0513 https://orcid.org/0000-0002-6621-2570 https://orcid.org/0000-0001-5571-8819 https://orcid.org/0000-0002-0118-4521 https://orcid.org/0000-0002-6445-3688

Objetivo: Descrever a experiência de trabalhadores/pesquisadores em realizar o Webinário: Enfermagem Obstétrica e sua força de trabalho em tempos de COVID -19: um retrato das ações no Brasil. Método: Relato de experiência sobre as reflexões a respeito da força de trabalho das Enfermeiras Obstétricas representadas pelas cinco regiões do Brasil no Webinário das ações da ABENFO nacional. Resultados: A experiencia demonstrou a força de trabalho de Enfermagem Obstétrica articulada aos direitos a saúde das mulheres em fase reprodutiva em tempos de COVID-19 a partir do pensamento crítico, a comunicação, a colaboração e a criatividade. Conclusão: É fundamental e necessária a interação, mediante o uso das tecnologias disponíveis, entre os profissionais da enfermagem obstétrica de todas as regiões do país, a fim de compartilhar experiências e encontrar novas possibilidade para o cuidado de mulheres no ciclo gravídico puerperal neste momento de crise.

Descritores: Enfermagem Obstétrica; Infecções por Coronavírus; Direitos da Mulher.

\section{OBSTETRIC NURSING AND ITS WORK FORCE IN COVID-19 PANDEMIC: EXPERIENCE REPORT FROM BRAZILIAN REGIONS}

Objective: To describe experience of workers/researchers in performing the Webinar: Obstetric Nursing and its workforce in COVID-19 pandemic: a portrait of actions in Brazil. Method: Experience report on there flections regarding the work force of Obstetric Nurses represented by five regions of Brazil in Webinar of the actions of the Brazilian Association of Obstetrics and Nurses Obstetricians. Results: The experience demonstrated the work force of Obstetric Nursing articulated to the women's health rights in reproductive phase in COVID-19 pandemic from critical thinking, communication, collaboration, and creativity. Conclusion: The need for interaction, through the use of available technologies, among obstetric nursing professionals from all regions of the country is fundamental, in order to share experiences and find new possibilities for the care of women in pregnancy and postpartum a tthis time of crisis.

Descriptors: Obstetric Nursing; Coronavirus Infections; Women's Rights.

ENFERMERÍA OBSTÉTRICAYSU FUERZADE TRABAJO EN ELTIEMPO COVID-19: INFORME DE EXPERIENCIADE LAS REGIONES BRASILEÑAS Objetivo: Describir la experiencia de los trabajadores / investigadores en la realización del seminario web: Enfermería obstétrica y su fuerza laboral en tiempos de COVID -19: un retrato de las acciones en Brasil. Método: Informe de experiencia sobre las reflexiones acerca de la fuerza laboral de enfermeras obstétricas representadas por las cinco regiones de Brasil en el seminario web nacional de acciones ABENFO. Resultados: La experiencia demostró la fuerza laboral de Enfermería Obstétrica articulada a los derechos de salud de las mujeres en edad reproductiva en tiempos de COVID-19 desde el pensamiento crítico, la comunicación, la colaboración y la creatividad. Conclusión: Es fundamental y necesaria la interacción, a través del uso de las tecnologías disponibles, entre profesionales de enfermería obstétrica de todas las regiones del país, para compartir experiencias y encontrar nuevas posibilidades para el cuidado de las mujeres en el ciclo de embarazo puerperal en este momento de crisis.

IEscola de Enfermagem Aurora de Afonso Costa da Universidade Federal Fluminense, Niterói, RJ,Brasil.

${ }^{2}$ Escola de Enfermagem da Universidade Federal de Minas Gerais, Belo Horizonte, MG, Brasil.

${ }^{3}$ Hospital das Clinicas da Universidade Federal de Minas Gerais, Belo Horizonte, MG, Brasil

${ }^{4}$ Escola de Enfermagem da Universidade Federal do Rio Grande do Sul, Porto Alegre, RS, Brasil.

${ }^{5}$ Faculdade de Enfermagem da Universidade Federal de Mato Grosso, Cuiabá, MT, Brasil.

${ }^{6}$ Centro de Ciências da Saúde, Faculdade de Enfermagem da Universidade Federal da Paraiba, João Pessoa, PB, Brasil.

${ }^{7}$ Fundação Santa Casa de Misericórdia do Estado do Pará, Belém, PA, Brasil.

Autor Correspondente: Valdecyr Herdy Alves E-mail: herdyalves@yahoo.com.br

Recebido: 06/6/20 Aceito: 23/6/20 
INTRODUÇÃO

Em 30 de janeiro de 2020, a Organização Mundial da Saúde (OMS) declarou que o surto da doença causada pelo novo coronavirus (SARS-CoV-2) constituia-se em uma Emergência de Saúde Pública de importância Internacional. Em 11 de março de 2020, a COVID-19 foi caracterizada pela OMS como uma pandemia, neste momento eram confirmados no mundo 3.759 .967 pessoas contaminadas e 259.474 mortes por COVID-19. No Brasil, neste mesmo dia o Ministério da Saúde enunciava 52 casos confirmados, 907 suspeitos e nenhum óbito1.

A crise mundial disparada pelo COVID-19 pode colocar sistemas de saúde em colapso. No Brasil, em especial na força de trabalho dos profissionais de saúde que apresentam inúmeros problemas estruturantes inviabilizando processos de trabalho a partir das concentrações geográficas e institucionais; polarização entre as categorias profissionais de saúde; jornadas de trabalho e as questões de gênero que revela desigualdades históricas².

As repercussões da COVID-19 tanto para as mulheres, para os recém-nascidos, suas famílias e comunidades, quanto para as equipes de Enfermagem, enfermeiras obstétricas, técnicos e auxiliares que atuam na linha de frente, no contexto da atenção obstétrico-neonatal em tempos de pandemia, ainda são limitadas. No entanto, sabe-se que surtos como estes podem afetar a saúde e ameaçar os direitos sexuais e os direitos reprodutivos, no nível individual e coletivo, com implicações na dinâmica e organização dos serviços de saúde, bem como na vida das pessoas ${ }^{3}$.

Na perspectiva de ofertar apoio à força de trabalho da Enfermagem Obstétrica, a Associação de Obstetrizes e Enfermeiros Obstetras (ABENFO) e suas Seccionais reconhecem o papel relevante da Enfermagem em tempos de pandemia e as relações instituidas no/com o processo de produção em saúde, reafirmando e fortalecendo a unidade, identidade/ multiplicidade e autenticidade que, se a coloca em um lugar protagônico, também the confere desafios permanentes. Além disso, melhorias na legislação acerca da carga horária, valorização e definição do piso salarial, e condições dignas de trabalho pairam como necessidades urgentes.

Neste cenário de luta, instabilidade e afastamento social, imposto pela pandemia, o uso de plataformas digitais possibilitou ABENFO e COFEN, Webinário: com objetivo de promover e valorizar o aprimoramento técnico-científico, ético-político, baseado nas evidências científicas, nas políticas públicas nacionais e internacionais e no saber-fazer da Enfermagem Obstétrica, visando à qualificação da atenção obstétrico neonatal e sua contribuição no fortalecimento do SUS e em defesa dos direitos das mulheres, em tempos de COVID-19.
Assim este artigo tem o objetivo de relatar a experiência de trabalhadores/pesquisadores em realizar o Webinário: Enfermagem Obstétrica e sua força de trabalho em tempos de COVID -19: um retrato das ações no Brasil.

\section{MÉTODO}

\section{Tipo de Estudo}

Trata-se de um relato de experiência produzido a partir das ações da ABENFO e suas Seccionais em parceria como a Comissão de Saúde e da Mulher do COFEN, desenvolvidas no Webinário que abordava a força de trabalho das Enfermeiras Obstétricas no enfrentamento da pandemia da COVID-19 nas cinco regiões do Brasil.

\section{Sujeitos envolvidos na experiência}

As reflexões dizem respeito as experiencias dos autores/ organizadores, das cinco regiões do país em realizar o Webinário: Enfermagem Obstétrica e sua força de trabalho em tempos de COVID -19: um retrato das ações no Brasil.

\section{Periodo de Realização da Experiência}

O webinário foi realizado no período de 05 de maio de 2020 no horário de 18:00 às 21:30, utilizado o aplicativo de videoconferência Zoom.

\section{Cenário do Estudo}

A partir da declaração do estado de emergência sanitária no país, as reuniões da ABENFO passam a ser realizadas virtualmente, e com prioridade para as ações pactuadas com a International Confederation of Midwives (ICM), a qual a ABENFO vincula-se como associação membro, de modo que emergiu a necessidade de conhecer e discutir as experiências das profissionais da área no contexto do COVID-19. O conteúdo do webinário foi organizado a partir da seguinte pergunta norteadora: como atua a força de trabalho da Enfermagem Obstétrica no enfrentamento da pandemia de COVID-19 nas regiões do Brasil?

A coleta de informações foi realizada virtualmente e mediante contato telefônico e se estabeleceu num processo de análise crítico-reflexivo e prática, a partir das experiências no atual cenário de parto e nascimento, dos relatos de profissionais em posição de liderança na ABENFO e da literatura atualizada em relação à COVID-19.

A condução do seminário foi realizada pelos autores deste relato, apresentando ações da América Latina e Caribe, representado pela ICM South América; região Norte, representada pela Seccional - ABENFO PA; região Sul, representada pela Seccional - ABENFO RS; região Centro-Oeste, representada pela Seccional - ABENFO MT; região Sudeste, representada pela Seccional - ABENFO MG; região Nordeste, representada pela Seccional - ABENFO PB e ABENFO Nacional. 


\section{OBJETIVO DA EXPERIÊNCIA}

Compartilhar reflexões da experiência de realização de um Webinário da Enfermagem Obstétrica e sua força de trabalho em tempos de COVID -19: um retrato das ações no Brasil.

\section{DESCRIÇÃO DA EXPERIÊNCIA}

Além das apresentações das autoras representantes de cada região do Brasil, o Webinário trouxe também a experiência da força de trabalho da enfermagem obstétrica no enfretamento da pandemia.

\section{Região Sudeste}

A região sudeste composta pelos estados de São Paulo $(\mathrm{SP})$, Rio de Janeiro (RJ), Espírito Santo (ES) e Minas Gerais (MG) possui o maior número de casos confirmados da COVID-19 de todo Brasil. Essa região acumula 70.342 casos e 5.723 óbitos $^{4}$. Da mesma forma, predomina o maior número de profissionais de enfermagem com 4755 casos (suspeitos/ confirmados) e 35 óbitos $^{5}$. A força de trabalho em saúde desta região tem dispendido esforços na construção das notas técnicas, recomendações, pesquisas e produções de conhecimento para o momento desafiador.

No estado de SP, a ênfase foi dada à organização de um hospital de referência na capital que isolou o seu maior instituto, destinando metade dos leitos da unidade para o tratamento das vítimas de COVID-19. Na capital, dois hospitais de campanha estão em funcionamento e auxiliam no atendimento dos pacientes com diagnóstico de doença. Também foi criada uma rede de triagem com cinco centros, em conjunto com a prefeitura de São Paulo, para atendimento aos casos suspeitos do COVID-19.

Em MG foram implantadas10 unidades sentinelas na capital do estado e um hospital de campanha na região metropolitana para atendimento dos casos de COVID-19. Também foram organizados hospitais de referência para o atendimento às gestantes e parturientes com a doença nos estados de MG, RJ, e no ES.

\section{Região Nordeste}

A situação do nordeste compostas pelos estados do Maranhão (MA), Piauí (PI), Ceará (CE), Rio Grande do Norte (RN), Pernambuco (PE), Paraíba (PB), Sergipe (SE), Alagoas (AL) e Bahia (BA) retrata as desigualdades do país, pois possui grande extensão territorial e acesso limitado a serviços e tecnologias em saúde. A região apresenta 52.700 casos e 3.167 óbitos, com taxa de letalidade variando entre as capitais de 2,2\% em Sergipe (menor taxa) a 12,8\% em Pernambuco (maior taxa) por COVID-194

Essa região do país amarga o 2으 lugar em número de casos 1512 (suspeitos/confirmados) e 13 óbitos ${ }^{5}$. Recortando, para a enfermagem obstétrica, os dados coletados por formulário eletrônico apontaram para afastamentos por suspeita, confirmação ou grupo de risco. A Bahia (18 EOS afastadas), Maranhão (16 EOS afastadas) e Pernambuco (11 EOs afastadas) são os estados que sofreram maior impacto no momento.

No tocante à rede de atenção, os estados possuem planos de contingência para o enfrentamento do COVID-19, com notas técnicas estaduais com correspondência às notas do Ministério da Saúde, e maternidades organizadas para receber mulheres com suspeita e/ou confirmação da doença. $\mathrm{Na} \mathrm{Pa}$ raiba, a maternidade de referência para COVID-19 localiza-se na capital. Nela foi aberto o ambulatório de egressas, conduzido por enfermeiras obstétricas que fazem acompanhamento por telefone das mulheres que foram infectadas, até o 140 dia de alta, avaliando a evolução dos casos e a necessidade de retorno

Os estados de AL, MA e PI também organizaram serviços de referência para o atendimento das mulheres no ciclo gravídico puerperal com COVID-19, ambos localizados na capital de cada estado. O Estado da Bahia, possui a maior cobertura em maternidades de referência para COVID-19 da região, com 03 serviços de referência.

\section{Região Sul}

O Sul do Brasil, em seus três estados, Paraná (PR), Rio Grande do Sul (RS) e Santa Catarina (SC), acumula 7.706 casos e 273 óbitos por COVID-194, e os casos em profissionais de enfermagem totalizam 1054 (suspeitos/confirmados) e 2 óbitos ${ }^{5}$. Observa-se diferenças importantes entre os estados, uma vez que o PR tem muitos menos casos notificados quando comparado ao RS e SC, no entanto, apresenta taxa de mortalidade alta, que deve haver subnotificação neste estado.

Historicamente esta região é caracterizada pela dificuldade em avançar em práticas de humanização no parto/nascimento e por adotar, na contramão das políticas e evidências científicas, modelo de atenção medicalizado. Avanços na humanização obstétrica, obtidos com a Rede Cegonha, estão sendo questionados em algumas cidades da região no enfrentamento da pandemia. No PR houve o fechamento de duas maternidades de referências para o atendimento do parto de risco habitual, onde assistência era conduzida por enfermeiras obstétricas. Os serviços foram reorganizados para atendimento exclusivo da pandemia, e os partos foram deslocados para hospitais gerais.

Situação correspondente aconteceu em Porto Alegre, capital do RS, onde foi anunciado o fechamento da maternidade de referência para assistência materno e infantil, com transferência dos atendimentos para um hospital municipal com limitações importantes de recursos físicos e humanos, e nas práticas humanizadas.

Nas duas situações os trabalhadores das maternidades 
não foram ouvidos. Embora, em Curitiba lutassem manter o modelo humanizado e as de Porto Alegre, em consonância com as políticas públicas, se reinventavam para fortalece-lo em serviços aderidos ao Projeto ApiceON. Além disso, destaca-se a, contratação de $2 / 3$ a mais de enfermeiras obstétricas nesses serviços. A enfermagem obstétrica luta para garantir às mulheres o direito de parir com segurança e dignidade, no Sul do Brasil.

\section{Região Centro Oeste}

A região Centro-Oeste constituída pelos estados do Mato Grosso (MT), Mato Grosso do Sul (MS), Goiás (GO) e o Distrito Federal (DF), responde por 3,0\% dos casos de COVID-19 no Brasil, o que corresponde a 4.656 casos e 118 óbitos $^{4}$, sendo a menos afetada no país até o momento. Contudo, especificidades locais, como a vasta extensão territorial e o número limitado de leitos por habitantes de MT, as fronteiras com outros países e as reservas indígenas do MS, bem como a proximidade com polo político do DF e de GO, relacionadas com a rápida expansão da pandemia, apontam para potenciais fragilidades do estado no enfrentamento da doença.

Os casos de profissionais de enfermagem totalizam 201 (suspeitos/confirmados) e 2 óbitos por COVID-195. O número de profissionais da enfermagem afastados por suspeita/confirmação, e por pertencerem a grupos de risco para doença também é elevado na região, e a participação de residentes em enfermagem obstétrica, tem sido essencial na composição da atuação da enfermagem e para a qualidade do cuidado obstétrico nos serviços de saúde da região.

Todos os estados elaboraram notas técnicas para a atendimento às gestantes, parturientes, puérperas e ao RNs durante a pandemia COVID-19, tendo como referência as recomendações do Ministério da Saúde. Muitos serviços desenvolveram protocolos institucionais a partir da realidade de local, fato que gera certa disparidade do desenvolvimento do cuidado obstétrico, a depender de recursos humanos e estruturais de cada serviço, que tanto potencializam como limitam o desenvolvimento das Boas Práticas em tempos de pandemia.

Também foram organizados serviços de referência para o atendimento especializado de mulheres no ciclo gravídico-puerperal suspeitas/confirmadas com COVID-19 em todas os estados da região Centro-Oeste. Esta organização gerou a mudanças nos fluxos de vinculação das gestantes à maternidade de referência, e preocupações no que tange ao acesso destas mulheres ao serviço de saúde quando necessário. 0 cuidado pré-natal de risco habitual mantém os atendimentos conforme preconizado pelo MS, com as adaptações recomendadas, atividades coletivas presenciais foram suspensas. No DF alguns serviços estão ofertando acompanhamento on- -line, em complementação às consultas presenciais.

\section{Região Norte}

A região Norte - Amazonas (AM), Roraima (RR), Amapá (AP), Pará (PA), Tocantins (TO), Rondônia (RO), Acre (AC), apresenta 27.195 casos e 1.844 óbitos por COVID-194. Os casos de profissionais de enfermagem contaminados totalizam 454(suspeitos/confirmados) e 06 óbitos por COVID-19.Há também número significativo de profissionais da área afastados por suspeita de COVID-195.

O estado do AM é o mais acometido na região com 7.242 casos confirmados, e 584 mortes. Além disso o estado possui sistema de saúde frágil, com número insuficiente de serviços/leitos e de profissionais de saúde para a atendimento das pessoas com a doença. Na recomposição deste quadro foram contratados 267 profissionais de saúde pelo projeto "O Brasil conta comigo". O estado do PA apresenta situação semelhante, ambos estão à beira do colapso do sistema de saúde e avaliando a imposição de medidas mais severas de isolamento social ${ }^{4}$.

Cada estado possui uma maternidade de referência, e os casos de mulheres gestantes com sintomas respiratórios são avaliados e, quando necessário, encaminhados para os hospitais de retaguarda para a Covid-19, o mesmo acontece com casos graves que são encaminhados via central de regulação para estes serviços.

A região apresenta expressivo contingente de populações indígenas. As recomendações baseadas nas boas práticas na gestação, no parto, nascimento e puerpério estão mantidas para todas as mulheres que não tenham sinais e sintomas ou diagnóstico confirmado do COVID-19, ou para aquelas consideradas curadas dessa doença. Os estados estimulam a elaboração de protocolos para o atendimento das mulheres com diagnóstico de COVID-19, e reforça a importância de manter o acompanhamento pré-natal, através de agendamentos com horários pré-estabelecidos.

Em Rondônia (RO) instalaram um sistema de call-center para identificação de casos suspeitos de COVID-19 e orientações aos usuários. Neste canal, enfermeiros e médicos orientam os cuidados que devem ser tomados e quando há necessidade de isolamento e de procurar por atendimento nos serviços de saúde.

\section{PRINCIPAIS RESULTADOS ALCANÇADOS}

Se reinventar deve ser a tônica do momento. Seja no âmbito pessoal eu/ou, profissional. As mudanças diante da pandemia nos obrigam a análises das ações de enfermagem obstétrica e sua força de trabalho. Para isso, é necessário aprender e/ou melhorar o exercício do pensamento crítico, 
a comunicação, a colaboração e a criatividade. A flexibilidade mental e o equilibrio emocional definirão nossa adaptação aos novos aprendizados.

A experiência de trabalhadores/pesquisadores em organizar e realizar o Webinário sobre a força de trabalho da enfermagem obstétrica no enfrentamento da pandemia, identificou que o ensino e o aprimoramento profissional contínuo são necessários e podem ser facilitados por plataformas digitais disponibilizadas por instituições e organizações em prol de um bem coletivo de distanciamento social.

Atividades online, cursos gratuitos disponíveis, reuniões remotas, bem como novos modos de ser/estar no trabalho podem ser espaços de educação permanente e de aprimoramento contínuo em relação ao cuidado às mulheres e recém-nascidos. Distantes fisicamente, mas ainda mais conectados socialmente com a utilização da ferramenta da rede da internet tornou-se a realidade nesse tempo de pandemia do COVID-19.

Conforme recomendado pelo MS na Nota Técnica, que trata da infecção COVID-19 e os riscos às mulheres no ciclo gravídico-puerperal ${ }^{6}$, o cuidado obstétrico recomendado pela Rede Cegonha ${ }^{7}$, deve ser preservado e incentivado para suprir da melhor forma possivel as necessidades assistenciais às gestantes, puérperas e recém-nascidos. Entretanto, as recomendações ministeriais precisam avançar no sentido de descrever o modelo obstétrico que podemos oferecer neste momento.

A força de trabalho da enfermagem obstétrica se destaca neste contexto, na medida em que historicamente se ocupa de garantir e potencializar os direitos das mulheres, e de prover cuidados humanizados e respaldados em evidências científicas. Especialmente neste período a categoria deve colaborar incondicionalmente no enfrentamento da pandemia e na luta pela garantia das boas práticas obstétricas e neonatais, com foco naquelas que ainda possuem avanços limitados e/ou não foram plenamente implementadas. Estas em tempos de crise como este são as mais passiveis de retrocesso ${ }^{8}$, convergindo para a necessária ampliação de redes que se expandam ao entono da Enfermagem potencializando sua ação protagônica?.

Outro aspecto de destaque na experiência foi a discussão das questões voltadas a saúde, trabalho e segurança dos profissionais da enfermagem obstétrica, composta majoritariamente por mulheres, que cuidam de outras mulheres, e que impõem a necessidade de vozes a essas trabalhadoras da saúde em meio à pandemia e suas relações sociais, financeiras e políticas.

O modelo de cuidado e formas de cuidar também foram temas de reflexão e discussão, na medida em que a pandemia nos leva a questionar se o modelo obstétrico ofertado no país, centrado a assistência hospitalar, é de fato o mais adequado e seguro para as mulheres e seus filhos. Novas possibilidades de cenários e de cuidado precisam ser experimentadas para proteger o binômio mãe-filho da infecção COVID-19

Obtivemos um público de 169 pessoas nesse webnário que se manifestaram com perguntas, opiniões, sugestões por meio do chat. Parabenizaram e recomendaram a manutenção do canal de comunicação. Apontaram a preocupação acerca em ampliar a testagem das gestantes, e ainda com os impactos da pandemia na morbimortalidade materna e neonatal no país.

\section{Limitações da experiência}

A principal limitação do estudo está relacionada com a falta de documentos que demonstrasse os fluxos assistenciais no campo da obstetrícia, em tempos de COVID-19, bem como os registros de profissionais da área e de mulheres no ciclo gravídico-puerperal acometidos pela doença.

\section{Considerações para a prática}

Indo ao encontro das diversas estratégias de enfrentamento da pandemia, torna-se fundamental destacar a necessidade de interação, mediante o uso das tecnologias disponiveis, entre os profissionais da enfermagem obstétrica de todas as regiões do país, afim de compartilhar experiências e encontrar novas possibilidade para o cuidado de mulheres no ciclo gravídico puerperal neste momento de crise, em que é um grande desafio preservar as Boas Práticas para Parto e Nascimento e os direitos sexuais e reprodutivos das mulheres mantendo a segurança delas e dos profissionais de saúde.

\section{CONSIDERAÇÕES FINAIS}

A pandemia ocasiona alterações nos modos de cuidar das mulheres no ciclo gravídico puerperal, com repercussões tanto para quem cuida, como para quem é cuidado, demonstrando a necessidade de reorganização e reajustes das práticas, sem, contudo, abrir mão da humanização e da segurança do binômio mãe-filho e da defesa dos direitos das mulheres.

Contribuição dos Autores: Concepção e/ou desenho do estudo: Alves VH, Souza KV, Carmo JMA, Moretto VL, Teixeira RC, Freitas WMF, Souza ELC; Coleta, análise e interpretação dos dados: Alves VH, Souza KV, Carmo JMA, Moretto VL, Teixeira RC, Freitas WMF, Souza ELC; Redação e/ou revisão crítica do manuscrito: Alves VH, Souza KV, Carmo JMA, Moretto VL, Teixeira RC, Freitas WMF, Souza ELC; Aprovação da versão final a ser publicada: Alves VH, Souza KV, Carmo JMA, Moretto VL, Teixeira RC, Freitas WMF, Souza ELC. 


\section{REFERÊNCIAS}

1 - Organização Pan-Americana de Saúde (OPAS). Folha informativa - COVID-19 (Doença causada pelo novo Coronavirus) [Internet]. 2020 [cited 2020 May 03]. Available from: https://www.paho.org/bra/index.php?option=com_content\&view=article\&id=6101:covid19\&Itemid=875.

2 - Mamede MV. Força de trabalho de enfermagem e obstetrícia e os novos Objetivos de Desenvolvimento Sustentável (2016-2030). Rev Rene [Internet]. 2017 [cited 2020 May 29]; 18(6): 710-711. Available from: http:// periodicos.ufc.br/rene/article/view/31068/71659

3 - Hussein J. COVID-19: What implications for sexual and reproductive health and rights globally?, Sexual and Reproductive Health Matters [Internet]. 2020 [cited 2020 May 11]; 28(1):1-3. Available from: https://www. tandfonline.com/doi/full/10.1080/26410397.2020.1746065.

4 - Ministério da Saúde. (BR). Painel Coronavirus [Internet]. 2020. [cited 2020 May 11]. Available from: https:// covid.saude.gov.br/.

5 - Conselho Federal de Enfermagem. Observatório da Enfermagem [Internet]. 2020 [cited 2020 May 04]. Available from: http://observatoriodaenfermagem.cofen.gov.br/.

6 - Governo Federal (BR). Nota Técnica no 12/2020. Dispõe sobre a Infecção COVID-19 e os riscos às mulheres no ciclo gravidico-puerperal. Diário Oficial da União. [Internet]. 2020 [cited 2020 Jun 03].

Available from: https://central3.to.gov.br/arquivo/505116/

7 - Ministério da Saúde (BR). Portaria n. 1.459, 24 junho 2011 [Internet]. 2011[cited 2020 Jun 03]. Available from: https://bvsms.saude.gov.br/bvs/saudelegis/gm/2011/prt1459_24_06_2011.html

8 - Souza KV, Schneck SC, Pena ED, Duarte ED, Alves VH. Direitos humanos das mulheres no parto frente à pandemia de Covid-19: o que fazer da enfermagem obstétrica. Cogitare Enferm [Internet]. 2020 [cited 2020 Jun 11]; 25: e73148.

Disponivel em: http://dx.doi.org/10.5380/ce.v25i0.73148.

9 - Santos Filho S, Souza KV. Metodologia para articular processos de formação-intervenção-avaliação na educação profissional em enfermagem. Ciênc. saúde coletiva [Internet]. 2020 [cited 2020 Jun 11]; 25(1): 7988. Available from:

http://dx.doi.org/10.1590/1413-81232020251.28322019 